\title{
Desarrollo de la comunicación gestual intencionada en bebés: Estudio de un caso
}

\section{Development of intentional communication trough gestures in infants: A case study}

\author{
Chamarrita Farkas ${ }^{1 *}$ \\ Pontificia Universidad Católica de Chile \\ (Recepción: Mayo 2007 - Aceptación: Junio 2007)a
}

\begin{abstract}
Resumen
Este artículo da cuenta del seguimiento de un bebé durante su primer año de vida, cuya madre fue entrenada en el desarrollo de gestos simbólicos. Se documenta el desarrollo inicial de este tipo de comunicación; su inicio, orden de aparición de los gestos, forma de adquisición, frecuencia, funciones y categorías. Se aprecia que todos los gestos documentados ocurrían frecuentemente y eran usados de manera flexible para referirse a objetos reales, juguetes o imágenes. Estos datos apoyan la idea que estas conductas sensoriomotoras pueden considerarse como conductas nominativas tempranas con intención comunicativa y función simbólica, y consecuentemente, como comunicación preverbal. Finalmente se discuten sus implicaciones para investigaciones futuras.
\end{abstract}

Palabras claves: Desarrollo infantil, desarrollo del lenguaje, comunicación preverbal, gestos simbólicos.

\begin{abstract}
This article refers the first year of an infant whose mother was trained in the development of symbolic gestures. It documents the initial development of this type of communication; when it began, the order in which the gestures appeared, the form of acquisition, frequency, functions and categories. All the gestures documented occurred frequently and were used flexibly to refer to real objects, toys or pictures. This data supporting that sensory-motor behaviors can considered as early labeling, with communicative intention and symbolic function, and consequently can considered preverbal communication. Finally, it discusses their implications for future research.
\end{abstract}

Key words: Infant development, language development, preverbal communication, symbolic gestures.

La comunicación constituye un proceso central a través del cual se intercambian y construyen significados con otros. Ya en los primeros meses de vida los niños utilizan el lenguaje no verbal para manifestar sus necesidades, expresar sus sensaciones y sentimientos, y vincularse con otras personas así como con el mundo que les rodea. Progresivamente el lenguaje no verbal, junto con la capacidad de representación, posibilitará el desarrollo de distintos lenguajes como el corporal, musical o plástico (Mineduc, 2002).

Parte importante del desarrollo teórico, así como del estudio del lenguaje, se ha focalizado casi exclusivamente en el desarrollo del lenguaje verbal. Así, se consideraba que las primeras palabras del niño marcaban el inicio de la habilidad de representar simbólicamente conceptos y usar los símbolos para el propósito expreso de comunicarse con otros (Goodwyn, Acredolo \& Brown, 2000).

Esta mayor focalización en el desarrollo del lenguaje verbal se ha debido en parte a los postulados del psicólogo Jean Piaget, uno de los autores más conocidos por sus estudios del desarrollo cognitivo de los niños. Para Piaget, el niño pasaba inicialmente por un período sensoriomotriz en el cual se encontraba ligado al estímulo y se limitaba a procesar estimulación específica a cada modalidad

\footnotetext{
1* La elaboración de este artículo contó con el financiamiento otorgado por el Fondo Nacional de Desarrollo Científico y Tecnológico, FONDECYT, No 1060778.

a Artículo recibido en mayo del 2007 y aceptado en junio del 2007.

Correspondencia a: Chamarrita Farkas, Escuela de Psicología, Pontificia Universidad Católica de Chile. Avda. Vicuña Mackenna 4860, Macul, Santiago, CHILE. Teléfono: (2) 354-7067. Fax: (2) 354-4844. E-mail: chfarkas@uc.cl
} 
sensorial, no siendo capaz de procesar información acerca de objetos. Luego, a los 2 años de edad, surgía la capacidad simbólica; ésta recién le permitía poderse representar mentalmente símbolos, lo cual era necesario para la aparición del lenguaje (Piaget, 1965).

Actualmente, estudios en Psicología cognitiva han podido demostrar que la capacidad de formar representaciones mentales surge bastante más precozmente que lo que Piaget proponía. Perner (1994) afirma que ya al mes de vida el bebé es capaz de diferenciar entre objetos para la succión nutritiva (pecho), de objetos para la succión no nutritiva (chupete). La información oral crea en el bebé un "esquema” interno que representa al objeto en la boca (referente) en tanto que "nutritivo” (sentido).

Los psicólogos cognitivos contemporáneos coinciden en que el bebé es capaz desde muy temprano de construir representaciones y esta capacidad se va desarrollando por etapas. Entre el nacimiento y el año o año y medio de vida hay un nivel primario (o representaciones primarias) en el cual el niño representa aspectos del mundo en forma seria y literal. Estas representaciones tienen una relación semántica directa con una realidad y sólo le permite concebir la situación real. No tienen una modalidad específica y se limitan a representar lo presente perceptible (Leslie, 1987; Perner, 1994).

Luego, alrededor de los 10 meses, se da un nivel secundario al cual Leslie (1987) se refiere como "representaciones secundarias” y Perner (1994) como “modelos múltiples”. El bebé es capaz de considerar dos o más representaciones de manera simultánea, lo cual le posibilita interpretar las representaciones y concebir entonces distintas situaciones (pasado/futuro, real/simulado). El niño es capaz de trascender la realidad y simular, o jugar al “como si”. Ello le posibilita entonces el comprender que una palabra o un gesto, son referentes de un determinado objeto o situación.

Otro tema de interés en el estudio del desarrollo del lenguaje se refiere a los precursores de éste, específicamente los pasos a través de los cuales el bebé gradualmente empieza a usar símbolos arbitrarios para referirse a fenómenos del mundo real. Ya Werner y Kaplan proponían en 1963 (cit. en Goodwyn, Acredolo \& Brown, 2000) que el desarrollo de la habilidad representacional le implicaba al niño el tolerar gradualmente un mayor distanciamiento entre el símbolo y su referente. Así, un símbolo onomatopéyico (ej. "guau") indica una menor distancia con el referente que el símbolo arbitrario (ej. "ladrar”), el cual implica una mayor demanda cognitiva ya que el niño debe mantener mentalmente esta relación sin el apoyo del ambiente. Desde esta perspectiva, podemos comprender que un gesto simbólico o comunicativo presenta una menor demanda cognitiva en relación a la palabra (símbolo arbitrario), ya que la distancia con el referente es menor, siendo posible entonces que surja más tempranamente en el desarrollo del niño.

\section{Desarrollo de la Intención Comunicativa}

El desarrollo de la intención comunicativa -el querer comunicarle algo a un otro- es fundamental para la comunicación. Alrededor de las 6 semanas de vida aparece la "sonrisa social”, una de las primeras conductas reportadas como poseedoras de una cierta intención comunicativa con otra persona (Perner, 1994).

Otro fenómeno relevante es el de "referencia social” que alude a la capacidad del niño de percibir y responder a la orientación afectiva de otra persona respecto a objetos y eventos del ambiente. Éste ha sido observado tempranamente en bebés por Charman (1994) y en distintos estudios sobre cómo el bebé considera a la madre como referente y en el manejo del temor a los extraños (Sroufe, 2000).

Posteriormente el niño debe desarrollar la importante capacidad de comprender la importancia de la mirada de la madre. Inicialmente éste es capaz de dirigir la mirada en la misma dirección que ella (6 meses). Luego, entre los 9 y 14 meses comienza a señalar, y entre los 12 y 16 meses trata de controlar la mirada del adulto para atraer su atención, ya sea para obtener algo, o simplemente por el solo hecho de disfrutar las expresiones de atención de éste (Perner, 1994).

Antes de los 8 meses de edad el niño sólo es capaz de interactuar en situaciones cara a cara, es decir, en situaciones sin referentes externos. Entre los 8 y 12 meses de edad en cambio, y antes de que aparezca el juego simbólico, el niño es capaz de desarrollar acciones o gestos comunicativos para regular sus interacciones con los demás, en relación a objetos externos. Estas conductas han sido 


\section{DESARROLLO DE LA COMUNICACIÓN GESTUAL INTENCIONADA EN BEBÉS: ESTUDIO DE UN CASO}

denominadas como “comunicación intencional prelingüística” (Gómez, Sarriá \& Tamarit, 1993), o “gestos simbólicos”.

\section{Comunicación Gestual Desde Una Perspectiva Evolutiva}

Ya Piaget (1965) proponía que alrededor de los 10 meses de edad aparecían los "gestos de ejecución”, los cuales se refieren a acciones como el observar insistentemente un objeto para indicar que se le quiere, dirigir la atención de un adulto hacia un objeto dándoselo, o apuntar objetos para demostrar interés o necesidad.

Estos gestos se corresponderían con los gestos protodeclarativos y protoimperativos descritos por Premack y Woodruff (1978), quienes proponen que en esta etapa la comunicación gestual presenta dos dominios:

a) Gestos protoimperativos: los gestos se utilizan para hacer peticiones y consisten en pedir a una persona que realice una acción determinada en beneficio del niño.

b) Gestos protodeclarativos: los gestos se utilizan para mostrar objetos a los demás y consisten en llamar la atención de una persona sobre un objeto determinado o sobre un acontecimiento del entorno, exigiéndole solamente que le preste atención.

Estos gestos intencionales tempranos han sido denominados como gestos deícticos o de ejecución, y constituyen un claro avance sobre el actuar meramente sobre los objetos, pero todavía son primitivos en cuanto a la sofisticación de la representación, ya que el significado del referente es claro sólo si el observador sigue la trayectoria del gesto hacia su objetivo. Pero sí debe reconocerse que estos gestos constituyen un importante paso en el desarrollo simbólico y pavimentan el camino al aprendizaje del lenguaje verbal (Goodwyn, Acredolo \& Brown, 2000).

Posteriormente a los gestos deícticos, surgen los “gestos simbólicos”, que entregan un equivalente simbólico sencillo, ya que constituyen acciones físicas simples que pueden ser usadas para representar objetos y eventos, así como expresar deseos, necesidades, pensamientos y emociones. Tienen una función comunicativa y nominativa, conllevan su significado en su forma y sustituyen al habla, hasta que ésta sea posible (Goodwyn, Acredolo \& Brown, 2000).

Otro importante hito en el desarrollo de los gestos comunicativos se ha encontrado durante el período de los 3 a 5 años de edad. Se propone que a los 3 años de edad surge el desarrollo de los gestos “icónicos”, descritos por Nicoladis, Mayberry y Genesee (1999), los cuales cumplen una función de afirmar más que de nominar, y no sustituyen sino más bien acompañan al habla.

En el inicio de este período hay una fuerte tendencia en el niño a depender de una parte de su cuerpo para representar la herramienta (ej. usar su dedo como el cepillo de dientes). En cambio a los 5 años de edad la capacidad representacional del niño se ha complejizado hasta el punto que es capaz de entender o producir la acción relevante por sí misma, como si la herramienta estuviera allí (ej. mover la mano como si se estuviera sosteniendo un cepillo de dientes) (Boyatzis \& Watson, 1993; O’Reilly, 1995). En otras palabras, a los 5 años de edad el símbolo se ha distanciado del referente lo suficiente como para no necesitar aparentemente ningún símbolo concreto de la herramienta (Goodwyn, Acredolo \& Brown, 2000).

\section{Los Gestos Simbólicos}

El desarrollo de los gestos simbólicos se da en una variedad impresionante, lo cual es atribuido a que los adultos le modelan al niño no sólo los gestos en sí mismos, sino el uso de éstos como herramientas para la comunicación. Así, el niño aprende tanto la "forma” como la "función”, además del hecho que la forma del gesto debe permanecer constante para que la comunicación sea exitosa (Goodwyn, Acredolo \& Brown, 2000).

La comunicación gestual combina 3 elementos: La forma o configuración de la mano (u otra parte del cuerpo), el lugar en que el signo se forma y el movimiento que se hace. Es similar al lenguaje 
verbal en cuanto al uso de gestos arbitrarios, a los facilitadores del aprendizaje, a la forma en que se inicia y desarrolla, y por el tipo de errores que se cometen (Mehler \& Dupoux, 1994).

En el estudio del desarrollo espontáneo de estos gestos simbólicos durante el segundo año de vida se ha podido apreciar que estos gestos son bastante comunes en infantes entre los 10 y 24 meses de edad, período en el cual cumplen una función utilitaria hasta que los equivalentes verbales sean posibles, y pueden aprenderse a través de rutinas interactivas con los adultos, de la observación de modelos, o prestarse de las acciones realizadas con el objeto de referencia (Acredolo \& Goodwyn, 1985, 1988).

Este aprendizaje de los gestos se da en un momento en que en el lactante aparece una necesidad de comunicarse, acompañada de un gran desarrollo cognitivo. Está descubriendo todo un nuevo mundo así como su propio cuerpo, pero no puede comunicarlo porque no conoce las palabras. Esto puede apreciarse en el interés que demuestra por las cosas, en las miradas fijas que sostiene sobre sus padres, las cuales acompaña por sonidos insistentes o gestos, y en la frustración resultante cuando el adulto no comprende lo que trata de comunicarle. Si el padre no está atento a estas señales o no las comprende, finalmente el niño deja de intentar comunicarse por esta vía (Acredolo \& Goodwyn, 1988).

La habilidad para desarrollar estos gestos aparece más tempranamente que la habilidad para reproducir palabras (Acredolo \& Goodwyn, 2001), por tanto el aprendizaje de los gestos permite establecer un puente de comunicación con el niño, hasta la aparición del habla. Incluso cuando ésta aparece, no todas las palabras tienen el mismo nivel de dificultad, por lo que el gesto puede servir hasta que la palabra pueda ser dicha o comprendida; ello resulta en que el niño utiliza de manera simultánea palabras que ya conoce, así como gestos para aquellas palabras que aún no puede pronunciar.

Luego a medida que el niño va conociendo las palabras, ellas reemplazan al gesto, ya que son más sencillas (y más económicas) de ejecutar, no obstante no pierden su importancia dentro de la comunicación. Estudios realizados con preescolares ( 3 a 5 años de edad) demuestran que estas señales continúan jugando un importante rol en la comprensión del lenguaje en la medida que los niños desarrollan habilidades lingüísticas más complejas (Kelly, 2001).

Investigaciones indican que el inicio de la ejecución de estos gestos por parte del niño se da entre los 8 a 14 meses (Acredolo \& Goodwyn, 2001; Garcia, 1999; Moore, Acredolo \& Goodwyn, 2001), variando ampliamente de niño en niño, ya que en este aprendizaje influye el interés del niño por comunicarse, el desarrollo de las destrezas relacionadas (por ejemplo, memoria, imitación, atención) y la frecuencia con que los padres modelen estos gestos (Acredolo \& Goodwyn, 2001). Las primeras evidencias de su inicio se dan a través de conductas de atención conjunta, en las que el niño típicamente antes, durante o después de la realización del gesto, hace contacto ocular con el adulto (Gómez, Sariá \& Tamarit, 1993).

El niño puede desarrollar estos gestos a través de dos formas: en la primera, el lactante imita los gestos que le hacen los adultos -especialmente las figuras significativas-ya sea de manera intencionada o casual. En la segunda forma el niño crea sus propios gestos, los cuales deben ser comprendidos por el adulto. Ello implica que debe comprender la esencia de un objeto o situación, ya sea su cualidad principal o función, y transmitirla a través de una señal o gesto que la represente (Acredolo \& Goodwyn, 2001). Esta habilidad para crear espontáneamente sus propios gestos simbólicos como una forma de evadir los obstáculos que implican las demandas articulatorias del lenguaje verbal, ha sido observada tanto en estudios de casos, así como en estudios transversales y longitudinales (Acredolo \& Goodwyn, 1985, 1988).

\section{Beneficios del Desarrollo Intencionado de la Comunicación Gestual}

A través de numerosas observaciones, investigaciones y reportes de los padres, se ha podido demostrar que los niños que han sido entrenados en el aprendizaje de gestos aprenden a hablar más rápido, su desarrollo del lenguaje es más precoz, y su vocabulario -tanto de palabras como de gestos- es significativamente mayor (Acredolo \& Goodwyn, 2001; Moore, Acredolo \& Goodwyn, 2001; Tomasello \& Farrar, 1986). 


\section{DESARROLLO DE LA COMUNICACIÓN GESTUAL INTENCIONADA EN BEBÉS: ESTUDIO DE UN CASO}

En estudios comparativos se observó además que estos niños entendían más palabras, tenían un mayor vocabulario, y se involucraban en juegos más sofisticados que el grupo control. A la vez, consistentemente obtenían puntuaciones más altas en tests estandarizados sobre su desarrollo del lenguaje comprensivo y expresivo (Goodwyn \& Acredolo, 1993, 1998; Goodwyn, Acredolo \& Brown, 2000).

Además de sus beneficios en el desarrollo del lenguaje, se han reportado beneficios en el desarrollo cognitivo de los niños. En estudios de seguimiento se vio que niños de 7 y 8 años que habían aprendido a comunicarse a través de gestos cuando pequeños, obtenían en el WISC-III puntajes de CI significativamente mayores que los niños del grupo control, tanto en la prueba completa, así como en las escalas verbal y motora (Acredolo \& Goodwyn, 2000).

En un estudio realizado por Moore, Acredolo y Goodwyn (2001) se pudo apreciar que los niños que habían sido entrenados en el uso de los gestos simbólicos, iniciaban períodos de atención compartida con sus cuidadores de manera significativamente más frecuente que los grupos control, y utilizaban además estrategias significativamente más complejas para lograr este fin. Las autoras concluyen de su estudio que el efecto de los gestos simbólicos en el desarrollo verbal está mediado por lo menos en parte por el incremento en la efectividad del infante para iniciar períodos de atención compartida.

En relación a aspectos socioemocionales, se ha visto que los niños que han sido entrenados en la utilización de los gestos para comunicarse, se frustran y enojan menos, lloran menos, y no utilizan preferentemente los gritos como formas de llamar la atención (Acredolo \& Goodwyn, 2001). Ello puede entenderse si consideramos que alrededor de los 9 a 10 meses de edad el lactante es bastante capaz de saber lo que quiere, necesita o siente, pero todavía no puede transmitirlo con palabras ni hacerse entender, lo cual le genera frustración. Esta frustración la expresa a través del llanto, gritos, pataletas y mal humor. La posibilidad de comunicarse a través de gestos le permite transmitir de una manera sencilla y rápida sus necesidades, lo cual disminuye la frustración, y resulta tremendamente reforzante para él en la medida que el otro comprende lo que trata de decirle.

\section{Interpretación de estos efectos}

Los resultados del aprendizaje de una comunicación gestual temprana sobre el aprendizaje y desarrollo del lenguaje verbal, se fundamentan en que el niño que aprende a comunicarse a través de esta vía realiza una especie de "apresto” para el habla, y se encuentra más motivado a aprender a hablar. Además, las señales del niño generan un mayor lenguaje en los adultos, en el sentido que al usar estas señales para llamar la atención sobre algo, los adultos responden de manera natural a la situación con una gran cantidad de gestos y palabras apropiadas, y mientras más lenguaje el niño escucha, más rápidamente se da el proceso de adquisición del habla (García, 1999, 2002).

También debe considerarse el hecho de que a través del gesto el niño dirige la atención de sus padres hacia aquello que le fascina, más que sólo escuchar pasivamente el nombre que los padres le ponen a las cosas que ellos consideran que son importantes para el niño, y es bien sabido que es más fácil aprender aquellas cosas que son más interesantes y motivantes para uno. Además, el aprender estas señales resulta excitante para el lactante, incrementando su motivación para aprender mejores formas de comunicarse (Goodwyn, Acredolo \& Brown, 2000; Acredolo \& Gooldwyn, 2001).

En un estudio realizado por Tomasello y Farrar (1986), se pudo observar que el uso del nombre del objeto por parte de la madre para referirse a los objetos a los cuales el niño ya estaba atendiendo en forma previa, se correlacionaba positivamente con el tamaño del vocabulario del niño así como la velocidad del aprendizaje de las palabras, versus situaciones en las cuales la madre redirigía el foco de la atención del niño a otro objeto.

A través del uso de los gestos simbólicos el niño empieza a aplicar conceptos, sin tener que esperar a la aparición del habla. Cuando el niño usa exitosamente un gesto simbólico, nos está indicando que entiende el concepto o categoría o condición en la cual se basa el gesto, reconoce el conjunto de sonidos (palabra dicha por los padres) como equivalentes a su gesto, y que obviamente ha configurado la función simbólica ya que la aplica en su comunicación. Por tanto su problema para hablar se relaciona claramente con la parte articulatoria del lenguaje (Goodwyn, Acredolo \& Brown, 2000). 
También debe considerarse que en la medida que el niño puede comunicarse tempranamente de manera más efectiva con sus figuras significativas (padres, cuidadores y otros), se reducen las interacciones negativas y aumentan las interacciones positivas. El niño y sus padres se pueden entender mejor mutuamente, y por tanto disfrutan más el estar juntos, generándose lazos más estrechos entre ellos (Acredolo \& Goodwyn, 2001).

Tanto para los padres como para el niño, el poder comunicarse entre ellos los motiva a trabajar fuertemente de manera recíproca. Por un lado el niño estará atento a asociar acciones específicas con contextos específicos, y activamente imitará estas acciones. Por el otro lado los padres no sólo modelarán acciones relevantes al niño, sino que además lo retroalimentarán cuando use gestos para comunicarse (Goodwyn, Acredolo \& Brown, 2000).

En base a lo anteriormente presentado, y considerando que este tema ha sido desarrollado y estudiado en Estados Unidos principalmente, surge el interés de conocer cómo se daría el desarrollo inicial de la comunicación gestual en un bebé chileno, cuya madre hubiese sido entrenada previamente en el uso de gestos simbólicos. Así, un primer objetivo de este estudio es constatar a través del estudio de un caso, si efectivamente estos gestos pueden ser adquiridos por parte del niño y utilizados por él como parte de su comunicación, antes de la adquisición del habla. Estos resultados preliminares permitirán orientar estudios futuros con más niños, respecto a los beneficios que les reportaría para su desarrollo, considerando los beneficios observados en estudios extranjeros.

Un segundo objetivo se refiere a describir el desarrollo inicial de la comunicación gestual en este infante, identificando la edad de aparición de los primeros gestos simbólicos, su orden de aparición, la forma de adquisición y de expresión, así como describir su significado, frecuencia de ocurrencia y contextos en los cuales se manifiestan.

Esta información permitirá nutrir intervenciones futuras en las cuales pueda educarse de manera más específica a padres y educadores sobre el modo en que se desarrolla la comunicación gestual, considerando a la vez los aspectos propios a la cultura chilena. Ello permitirá que las intervenciones sean más efectivas y evitará así sentimientos de fracaso y de frustración.

\section{Método}

\section{Diseño}

Este estudio se llevó a cabo a través de un diseño longitudinal de tipo exploratorio. La variable manipulada fue el entrenar a la madre en el uso de gestos en la comunicación con su hija. Se realizó un seguimiento hasta los 12 meses de edad, con el objetivo de explorar el desarrollo de la comunicación gestual intencionada en el primer año de vida del bebé.

\section{Participante}

El sujeto de este estudio fue un lactante de sexo femenino, hija de madre primigesta, de nivel socioeconómico medio, quien participó en un seguimiento con su madre desde los 6 meses y hasta cumplir 12 meses de edad.

\section{Instrumento}

Pauta de Registro. Para elaborar esta Pauta se utilizó como referencia la Pauta propuesta por Acredolo y Goodwyn (“I’m a Baby Signs Baby”2), pero incorporando información específica de modo de cumplir con los requerimientos del estudio. Su objetivo era permitirle documentar a la madre los gestos que la bebé fuera desarrollando. Esta Pauta consta de tres partes: En la primera parte se encuentran las instrucciones y ejemplos para llenar la Pauta, en la segunda parte se va registrando la información de los gestos que van surgiendo (descripción, edad de inicio, forma de aprendizaje,

2 (C) Copyright Baby Signs ${ }^{\mathrm{TM}}$, Inc. 2001. 


\section{DESARROLLO DE LA COMUNICACIÓN GESTUAL INTENCIONADA EN BEBÉS: \\ ESTUDIO DE UN CASO}

contextos en que se da, comentarios) y en la tercera parte se incluye el registro de la aparición de gestos combinados (dos o más gestos).

Esta Pauta fue previamente revisada por un grupo de jueces para adecuar su redacción, vocabulario y comprensión. La información que entrega es descriptiva y sólo se analiza de manera cualitativa.

\section{Procedimiento}

A los 6 meses del bebé y de manera previa a iniciar el seguimiento, la madre de la niña fue entrenada en cómo desarrollar la comunicación gestual en su bebé; ello se llevó a cabo a través de dos reuniones explicativas con ella y entrega de material escrito y gráfico de apoyo. Se le indicó además que transmitiera estas indicaciones a otras personas significativas que interactuaran frecuentemente con el bebé.

Se le entregó a la madre la Pauta de Registro, en la cual debía ir registrando diariamente los gestos que el bebé iba realizando. La información de dicha pauta se iba cotejando en visitas quincenales, en las cuales se aclaraban dudas de la madre y se corroboraba que el gesto anotado realmente cumpliera los criterios para ser un gesto comunicativo simbólico (Acredolo \& Goodwyn, 1985), los cuales eran:

- Estabilidad. El gesto debía repetirse de manera estable en el tiempo.

- Coherencia. El gesto debía relacionarse siempre con el mismo objeto o situación.

- Especificidad. El gesto debía relacionarse con un solo concepto, y no presentarse frente a otros conceptos.

- Generalización. El gesto podía usarse de manera generalizada (ej. Para todos los tipos de perro, no sólo un perro específico).

\section{Análisis de datos}

El análisis de los gestos desarrollados por la bebé en el período observado se realizó cualitativamente en base a la información descriptiva arrojada por la Pauta de Registro. Estos gestos recopilados durante el período de observación se analizaron y codificaron considerando los siguientes aspectos:

- Edad de inicio.

- Frecuencia de gestos logrados, por mes.

- Nivel de complejidad. Los gestos observados fueron categorizados en 3 niveles de complejidad, considerando la familiaridad con el objeto o situación (ej. “perro” versus “flamenco”), la complejidad del concepto (ej. “comida” versus “feliz”), la parte del cuerpo involucrada (ej. cara versus dedos), y el nivel de coordinación requerido.

- La forma de aprendizaje de los gestos (imitación versus espontáneos).

- Nivel de generalización; desde ser específicos a una sola situación (ej. el pato amarillo plástico), a generalizar el gesto a diferentes situaciones (ej. el pato amarillo, un pato real, una foto de un pato en un libro).

- Funciones y categorías. Se consideraron las funciones nominativa versus afirmativa, y los gestos como sustituyendo versus acompañando al habla. Las categorías consideradas, basadas en el reporte de estudios previos (Acredolo \& Goodwyn, 1985, 1988), fueron: a) Animales (ej. perro, gato, caballo); b) Objetos (ej. teléfono, avión, libro); c) Requerimientos o acciones cotidianas (ej. dame, dónde está, comer); d) Emociones (ej. pena, rabia, alegría); y e) Adjetivos (ej. grande, pequeño, ruidoso). 


\section{Resultados}

\section{Inicio y Frecuencia}

De acuerdo al registro llevado por la madre, la producción del primer gesto simbólico propiamente tal fue observada a los 7 meses de edad ("no más”). En el período comprendido entre los 7 a 9 meses de edad la frecuencia de aparición de gestos es baja $(\mathrm{n}=2)$ mientras que en el período comprendido entre los 10 y 12 meses la frecuencia aumenta exponencialmente en 4 veces $(n=9)$. Así, del total de gestos reportados, la mayoría de ellos aparece entre los 10 y 12 meses de edad. El Gráfico No 1 muestra el crecimiento de vocabulario gestual en el período comprendido entre el mes y los 12 meses de edad, mientras que en la Tabla $\mathrm{N}^{0} 1$ se puede apreciar los gestos producidos por la niña según su inicio y descripción.

Gráfico No 1: Crecimiento de vocabulario gestual expresivo.

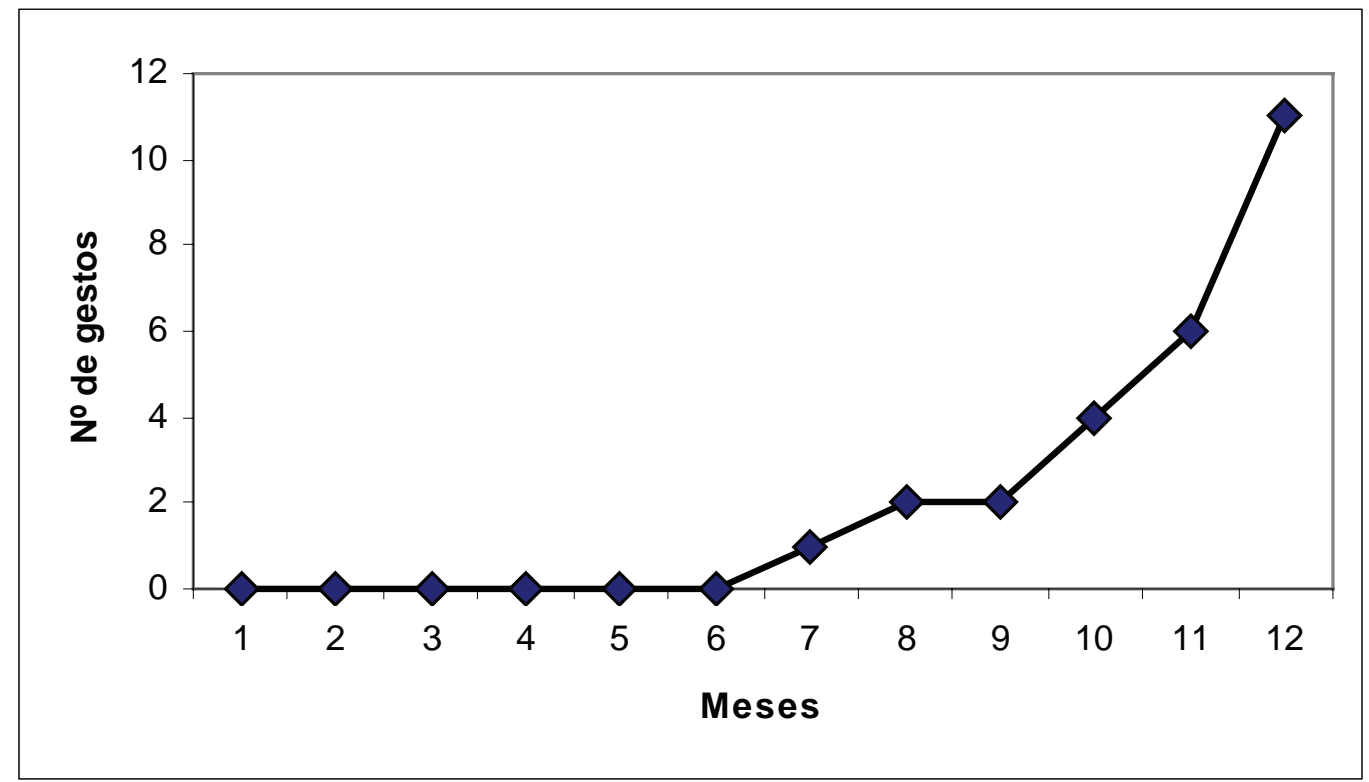

$$
\mathrm{N}=11
$$

Tabla No 1: Gestos producidos según inicio y descripción

\begin{tabular}{|c|c|c|c|}
\hline & Gesto & Edad de Inicio & Descripción \\
\hline 1 & “No más” & $7 \mathrm{~m}$. & La niña baja con fuerza brazos y piernas. \\
\hline 2 & “Perro” & $8 \mathrm{~m}$. & Saca la lengua y jadea. \\
\hline 3 & “Caballo” & $10 \mathrm{~m}$. & Junta y separa los labios, como tirando besos. \\
\hline 4 & "Ventilador" & $10 \mathrm{~m}$. & $\begin{array}{l}\text { Mira hacia arriba y sopla con la boca (se refiere a un } \\
\text { ventilador que hay en el techo). }\end{array}$ \\
\hline 5 & “Tómame” & $11 \mathrm{~m}$. & Estira los brazos hacia arriba, muy tiesos. \\
\hline 6 & “Ven” & $11 \mathrm{~m}$. & $\begin{array}{l}\text { Estira el brazo, abre y cierra la mano, con la palma hacia } \\
\text { arriba (acompañado de “mem, mem”). }\end{array}$ \\
\hline 7 & “Comer” & $12 \mathrm{~m}$. & Abre y cierra la boca varias veces. \\
\hline
\end{tabular}



8 "Beso"
$12 \mathrm{~m}$.
Coloca su mano frente a su boca, y al dar el beso, aleja la mano, como lanzándolo (acompañado de “mmaaa”).
9 "Chao/hola"
$12 \mathrm{~m}$.
Mano alzada, abierta, la mueve de lado a lado (acompa- ñado de "ao").
10 "Dame eso"
$12 \mathrm{~m}$.
En postura erguida, señala algo con el dedo (acompañado de "mía”).
11 "Pato"
$12 \mathrm{~m}$.
Abre y cierra los dedos de la mano, estirados, imitando un pico (acompañado de "cac" “cac").

\section{Nivel de Complejidad}

Los primeros gestos que produce la niña observada son de un nivel de dificultad menor, ya que requieren de poca coordinación y son fáciles de realizar (ej. “perro”, “caballo”; ambos se realizan con la boca, el primero sacando la lengua e imitando un jadeo y el segundo juntando y separando los labios varias veces), mientras que a medida que la lactante va creciendo los nuevos gestos van siendo más complejos e implican más requerimientos por parte de la niña para su ejecución (ej. "beso", apoya la mano abierta sobre su boca y al momento de producirse el beso mueve la mano hacia adelante, como lanzándolo).

Los movimientos realizados van desde movimientos bruscos, más globales y que requieren poco control, a movimientos más controlados y específicos, que requieren de la motricidad fina y de mayor coordinación.

Respecto a la zona del cuerpo involucrada, primero aparecen gestos que involucran la cara (especialmente la boca) y luego van descendiendo hacia brazos y manos (verticalmente el orden es descendente). A la vez, inicialmente los gestos involucran hombros y brazos, luego manos y finalmente dedos (horizontalmente, orden de centro a periferia).

Los primeros gestos involucran sólo una zona corporal (ej. inclinar hombros para referirse a "no está / desapareció / no sé”) y posteriormente comienzan a involucrar dos o más zonas (ej. llevarse la mano a la boca y tirar un beso, al despedirse). Finalmente, todos los gestos observados se refieren a conceptos familiares para la bebé. En la Tabla No 2 se puede apreciar la categorización de los gestos según su complejidad.

Tabla Nº 2: Descripción de los gestos producidos según nivel de complejidad

\begin{tabular}{|c|c|c|c|c|c|c|}
\hline & Gesto & $\begin{array}{l}\text { Edad de } \\
\text { Inicio }\end{array}$ & $\begin{array}{c}\text { Nivel de } \\
\text { familiaridad }\end{array}$ & $\begin{array}{l}\text { Complejidad } \\
\text { del concepto }\end{array}$ & $\begin{array}{c}\text { Parte corporal } \\
\text { involucrada }\end{array}$ & $\begin{array}{c}\text { Coordinación } \\
\text { requerida }\end{array}$ \\
\hline 1 & “No más” & $7 \mathrm{~m}$. & Alto & Bajo & Extremidades & Baja \\
\hline 2 & “Perro” & $8 \mathrm{~m}$. & Alto & Bajo & Boca & Baja \\
\hline 3 & “Caballo” & $10 \mathrm{~m}$. & Alto & Bajo & Boca & Baja \\
\hline 4 & "Ventilador" & $10 \mathrm{~m}$. & Alto & Bajo & Ojos, boca & Baja \\
\hline 5 & “Tómame” & $11 \mathrm{~m}$. & Alto & Medio & Brazos & Media \\
\hline 6 & “Ven” & $11 \mathrm{~m}$. & Alto & Medio & Brazo, mano & Media \\
\hline 7 & "Comer" & $12 \mathrm{~m}$. & Alto & Medio & Воса & Media \\
\hline 8 & "Beso" & $12 \mathrm{~m}$. & Alto & Medio & Mano, boca & Alta \\
\hline 9 & "Chao/hola" & $12 \mathrm{~m}$. & Alto & Medio & Mano & Media \\
\hline 10 & "Dame eso" & $12 \mathrm{~m}$. & Alto & Medio & Dedo & Media \\
\hline 11 & "Pato" & $12 \mathrm{~m}$. & Alto & Bajo & Dedos & Alta \\
\hline
\end{tabular}

Nivel de familiaridad: Alto, bajo; Complejidad: Bajo, medio, alto; Coordinación: Baja, media, alta. 


\section{Forma de Aprendizaje}

De los gestos reportados por la madre a través de la Pauta de registro dos tercios fueron aprendidos por imitación del adulto mientras que un tercio de ellos fueron creados por la bebé. Llama la atención que los gestos creados por la niña son más frecuentes al inicio (ej. “no más”), mientras que los gestos aprendidos por imitación del adulto son más frecuentes hacia el final del seguimiento (ej. "pato”). En la Tabla $\mathrm{N}^{\circ} 3$ se observa la categorización de los gestos según su forma de aprendizaje.

Tabla No 3: Descripción de los gestos producidos según forma de aprendizaje

\begin{tabular}{|c|c|c|c|}
\hline & Gesto & Edad de Inicio & Forma de aprendizaje \\
\hline 1 & "No más" & $7 \mathrm{~m}$. & Espontáneo \\
\hline 2 & “Perro” & $8 \mathrm{~m}$. & Imitación \\
\hline 3 & “Caballo" & $10 \mathrm{~m}$. & Imitación \\
\hline 4 & "Ventilador" & $10 \mathrm{~m}$. & Espontáneo \\
\hline 5 & “Tómame" & $11 \mathrm{~m}$. & Espontáneo \\
\hline 6 & “Ven” & $11 \mathrm{~m}$. & Espontáneo \\
\hline 7 & "Comer" & $12 \mathrm{~m}$. & Imitación \\
\hline 8 & "Beso" & $12 \mathrm{~m}$. & Imitación \\
\hline 9 & “Chao/hola” & $12 \mathrm{~m}$. & Imitación \\
\hline 10 & "Dame eso" & $12 \mathrm{~m}$. & Espontáneo \\
\hline 11 & "Pato" & $12 \mathrm{~m}$. & Imitación \\
\hline
\end{tabular}

Forma de aprendizaje: Creación espontánea de la niña versus imitación de la conducta del adulto

\section{Nivel de Generalización, Funciones y Categorías}

En un primer momento el gesto aparece de manera bastante específica frente al objeto o situación concreta, pero luego rápidamente comienza a generalizarse a objetos o situaciones similares (ej. ya a los 8 meses puede apreciarse que la niña emite el gesto de "perro" para referirse al animal real así como a un juguete con esa forma).

Además, inicialmente los gestos sólo cumplen una función nominativa y sustitutiva del habla, ya que ésta no ha aparecido aún. La bebé realiza los gestos frente a un objeto que le muestra la madre (ej. un perro de peluche), cuando desea llamar su atención frente a algo que ella ve (ej. un pájaro) o cuando quiere comunicarle algo a ella (ej. gesto de "comida” cuando tiene hambre). Luego al aparecer las primeras palabras, cerca del año de edad, los gestos pueden acompañarlas como una manera de complementar el habla o dar más énfasis a lo que se quiere comunicar (ej. decir “peyo” en vez de perro, en conjunto con el gesto).

Finalmente, durante el período de seguimiento, los primeros gestos que aparecen corresponden a categorías de animales y objetos familiares para la niña $(n=4)$ y con pedidos o acciones que forman parte de la vida cotidiana $(\mathrm{n}=7)$. Hasta el año de edad no se habían observado todavía gestos relativos a emociones o adjetivos. En la Tabla $N^{\circ} 4$ se aprecian las categorías a las cuales corresponden los gestos producidos por la niña. 


\section{DESARROLLO DE LA COMUNICACIÓN GESTUAL INTENCIONADA EN BEBÉS: ESTUDIO DE UN CASO}

Tabla No 4: Descripción de los gestos producidos según categoría

\begin{tabular}{|c|c|c|c|}
\hline & Gesto & Edad de Inicio & Categoría \\
\hline 1 & “No más” & $7 \mathrm{~m}$. & Pedido o acción cotidiana \\
\hline 2 & "Perro” & $8 \mathrm{~m}$. & Animal \\
\hline 3 & “Caballo" & $10 \mathrm{~m}$. & Animal \\
\hline 4 & "Ventilador" & $10 \mathrm{~m}$. & Objeto \\
\hline 5 & “Tómame” & $11 \mathrm{~m}$. & Pedido o acción cotidiana \\
\hline 6 & “Ven” & $11 \mathrm{~m}$. & Pedido o acción cotidiana \\
\hline 7 & “Comer” & $12 \mathrm{~m}$. & Pedido o acción cotidiana \\
\hline 8 & "Beso" & $12 \mathrm{~m}$. & Pedido o acción cotidiana \\
\hline 9 & "Chao/hola” & $12 \mathrm{~m}$. & Pedido o acción cotidiana \\
\hline 10 & "Dame eso" & $12 \mathrm{~m}$. & Pedido o acción cotidiana \\
\hline 11 & "Pato" & $12 \mathrm{~m}$. & Animal \\
\hline
\end{tabular}

Categorías: Objeto, animal, peticiones o acciones cotidianas, emociones, adjetivos.

\section{Discusión}

En este análisis de caso se pretendía observar y documentar el desarrollo inicial de la comunicación gestual en un bebé chileno cuya madre había sido entrenada previamente para incluir el uso de gestos en la comunicación con su hija.

A través del seguimiento realizado, se pudo constatar que el primer gesto simbólico propiamente tal era observado a los 7 meses de edad, lo cual es relativamente precoz al compararlo con el rango de 8 a 14 meses reportado por otros estudios internacionales (Acredolo \& Goodwyn, 2001; García, 1999; Moore, Acredolo \& Goodwyn, 2001). Al revisar esos estudios en detalle, se aprecia que la edad de inicio de la intervención se relaciona con la edad de inicio de los gestos, y ésta se realizaba incluso recién a partir de los 11 meses de edad de los niños, a diferencia de este estudio donde la capacitación a la madre se realizó a los 6 meses de edad de la bebé.

Es importante no capacitar a los padres demasiado temprano en el uso de los gestos, para que estos no se frustren esperando poder observar los primeros gestos de sus hijos, pero a la vez estos datos podrían sugerir que la capacitación en este tipo de comunicación podría realizarse más tempranamente a lo que se ha venido haciendo, tema que requiere de mayor estudio y con una muestra mayor de niños.

Otro resultado de este estudio es el crecimiento en forma exponencial del vocabulario gestual expresivo, lo cual se corresponde con el crecimiento del vocabulario verbal expresivo (Mehler \& Dupoux, 1994) así como con otros estudios de crecimiento de vocabulario gestual en niños expuestos intencionadamente a la comunicación gestual (Acredolo \& Goodwyn, 1985).

En cuanto al nivel de complejidad en el desarrollo de la comunicación gestual, se aprecia un incremento en el nivel de complejidad en los gestos con el paso del tiempo. Así, los gestos iniciales son más familiares, fáciles de realizar, requieren menos coordinación, involucran menos partes corporales y se focalizan principalmente en la cara. Posteriormente los gestos irán siendo menos familiares, requerirán de mayor coordinación, involucrarán movimientos corporales combinados e incluirán otras partes del cuerpo (principalmente manos y dedos). 
Esta mayor complejidad corporal "articulatoria” se relaciona con el desarrollo de la motricidad fina y coordinación propios de la etapa evolutiva, pero a la vez permite hacer un paralelo con la complejidad creciente que se va dando en la adquisición del habla (Mehler \& Dupoux, 1994).

$\mathrm{Al}$ considerar la forma en que los gestos analizados fueron aprendidos, dos aspectos llaman la atención. El primero hace alusión a la edad en que se observa el primer gesto espontáneo ("no más, 7 meses), para lo cual es necesario que el niño previamente comprenda la esencia de un objeto, ya sea su cualidad principal o función, y sea capaz de transmitirla a través de una señal o gesto que la represente (Acredolo \& Goodwyn, 2001), lo cual nos permite atisbar a los complejos procesos cognitivos que tan tempranamente ya son capaces de realizar los niños.

El segundo aspecto guarda relación con el resultado de que los gestos espontáneos observados eran más frecuentes al inicio y que luego estos iban disminuyendo y siendo reemplazados por gestos imitados del adulto. Ello permite hipotetizar que gran parte de la comunicación espontánea por parte de los niños, si no es captada y reforzada por los adultos, disminuirá y dejará de producirse. A la vez, los gestos ofrecidos como comunicación por el adulto a través del modelaje, resultan en una vía más fácil para interactuar. Pareciera ser que el niño rápidamente comprende que estos intentos comunicacionales espontáneos son más difíciles de comprender para el adulto, y que para lograr una comunicación más exitosa, es mejor aprender aquellos gestos que éste le modela. Este proceso de socialización de la comunicación gestual a la vez es similar al proceso de socialización de la adquisición del habla.

Otro tema a señalar es que los gestos adquiridos rápidamente son generalizados a distintas versiones de representación del concepto. Ello constituye una evidencia de que efectivamente antes del año de edad el niño desarrolla representaciones secundarias que hacen posible la construcción de conceptos; proceso que no puede apreciarse hasta que las palabras sean aprendidas y aplicadas correctamente por el niño, mientras que los gestos nos ofrecen una forma de comunicación que sí permite observar este desarrollo.

Al igual que lo planteado por la literatura respecto de las funciones de los gestos simbólicos (Goodwyn, Acredolo \& Brown, 2000), se pudo apreciar en este estudio que los gestos observados cumplían con una función nominativa y sustitutiva del habla. Además, durante el primer año sólo se observan gestos que corresponden a categorías más concretas (cognitivamente), específicamente categorías de objetos, animales, peticiones y acciones cotidianas. Ello es coherente con el proceso de adquisición de la comunicación así como con el desarrollo cognitivo del niño, y se corresponde con lo descrito en estudios similares realizados en Estados Unidos con un grupo de niños (Acredolo \& Goodwyn, 1988).

En síntesis, el desarrollo inicial de la comunicación gestual intencionada posee características similares al desarrollo inicial del habla, así como otras características vinculadas al desarrollo de la motricidad, y constituye una importante ventana al desarrollo cognitivo de los niños.

Estas características, similares a las reportadas por estudios en el extranjero, son importantes de considerar al momento de enseñarle a padres y educadores sobre las ventajas de fomentar este tipo de comunicación y de qué forma hacerlo, diseñando una secuencia gradual de los gestos a estimular que considere: a) Características del niño, como el desarrollo de su motricidad e interés por el mundo que le rodea; b) Características del contexto, de modo de conocer qué elementos resultarán más familiares para el niño, adaptándose además a las características de su propia cultura; y c) Características de los gestos, considerando su nivel de complejidad, área del cuerpo involucrada y categoría a la cual se refiere. Todo lo anterior permitirá desarrollar un programa de comunicación gestual intencionada que incorpore un adecuado proceso educativo y manejo de expectativas tanto de padres como de educadores, quienes sabrán qué esperar y cuándo, en el desarrollo de esta comunicación en los niños. 


\section{DESARROLLO DE LA COMUNICACIÓN GESTUAL INTENCIONADA EN BEBÉS: ESTUDIO DE UN CASO}

\section{Referencias}

Acredolo, L. \& Goodwyn, S. (1985). Symbolic gesturing in language development: A case study. Human Development, 28, 40-49.

Acredolo, L. \& Goodwyn, S. (1988). Symbolic gesturing in normal infants. Child Development, 59, 450-499.

Acredolo, L. \& Goodwyn, S. (2000). The long-term impact of symbolic gesturing during infancy on IQ at age 8. Paper presented at the meetings of the International Society for Infant Studies, July, Brighton, UK.

Acredolo, L. \& Goodwyn, S. (2001). Los gestos del bebé. Barcelona, España: Oniro.

Boyatzis, C. J. \& Watson, M. W. (1993). Preschool children's symbolic representation of objects through gestures. Child Development, 64, 729-735.

Charman, T. (1994). How understanding the role of affect will help to decide if SAM and ToMM are separable cognitive mechanisms. Current Psychology of Cognition, 13, 5, 569-574.

Garcia, J. (1999). Sign with your baby. U.S.A.: Northlight Communications.

Garcia, J. (2002). Overview. [en línea]. Disponible en <http://www.sign2me.com/about.htm\#Program>.

Gómez, J. C.; Sarriá, E. \& Tamarit, J. (1993). El estudio comparado de la comunicación temprana y la teoría de la mente: Ontogénesis, Filogénesis y patología. Siglo Cero, 24, (6), 149, 47-62.

Goodwyn, S. \& Acredolo, L. (1993). Symbolic gesture versus word: Is there a modality advantage for onset of symbol use? Child Development, 64, 688-701.

Goodwyn, S. \& Acredolo, L. (1998). Encouraging symbolic gestures: Effects on the relationship between gesture and speech. En J. Iverson \& S. Goldin-Meadows (Eds.) The nature and functions of gesture in children's communication. San Francisco, U.S.A.: Jossey-Bass.

Goodwyn, S.; Acredolo, L. \& Brown, C. (2000). Impact of symbolic gesturing on early language development. Journal of Nonverbal Behavior, 24, 81-103.

Kelly, S. (2001). Broadening the units of analysis in communication: Speech and nonverbal behaviors in pragmatic comprehension. Journal of Child Language, 28, 2, 325-349.

Leslie, A. M. (1987). Pretense and representation: The origins of “Theory of Mind”. Psychological Review, 94, 4, 412-426.

Mehler, J. \& Dupoux, E. (1994). Nacer sabiendo. Madrid, España: Alianza.

Mineduc (2002). Bases curriculares de la educación parvularia. [en línea]. Disponible en <http://www.mineduc.cl/documentos/parvularia/BasesCurriculares.pdf>.

Moore, B.; Acredolo, L. \& Goodwyn, S. (2001). Symbolic gesturing and joint atention: Partners in facilitating verbal development. Paper presented at the Biennial Meetings of Society for Research in Child Development, April.

Nicoladis, E.; Mayberry, R. I. \& Genesee, F. (1999). Gesture and early bilingual development. Developmental Psychology, 35, 514-526.

O’Reilly, A. W. (1995). Using representations: Comprehension and production of actions with imagined objects. Child Development, 66, 999-1010.

Perner, J. (1994). Comprender la mente representacional. Buenos Aires, Argentina: Paidós.

Piaget, J. (1965). El lenguaje y pensamiento del niño pequeño. Buenos Aires, Argentina: Paidós.

Premack, D. \& Woodruff, G. (1978). Does the chimpanzee have a theory of mind? The Behavioral and Brain Sciences, 4, 515-526.

Sroufe, A. (2000). Desarrollo emocional. México: Oxford.

Tomasello, M. \& Farrar, M. (1986). Joint Attention and early Language. Child Development, 57, 1454-1463. 